Fumi-ichiro Yamamoto ${ }^{a}$

Patricia D. McNeill ${ }^{\text {a }}$

Miyako Yamamoto ${ }^{\mathrm{a}}$

Sen-itiroh Hakomori ${ }^{\text {a }}$

Teresa Harris ${ }^{\mathrm{b}}$

W. John Judd

Robertson D. Davenport ${ }^{\mathrm{d}}$

The Biomembrane Institute and

University of Washington, Seattle, Wash.;

"Pacific Northwest Regional Blood Services,

Portland, Oreg.;

Departments of

- Immunohematology and

Pathology, The University of Michigan

Hospitals, Ann Arbor, Mich., USA

\title{
Molecular Genetic Analysis of the ABO Blood Group System: 1. Weak Subgroups: $A^{3}$ and $B^{3}$ Alleles
}

\begin{abstract}
We have determined the nucleotide sequences of the coding region in the last two coding exons of $\mathrm{ABO}$ genes (which occupy $91 \%$ of the soluble form of $\mathrm{A}^{1}$ transferase) from 7 individuals with weak subgroup phenotypes. Four of the individuals had an $A_{3}$ phenotype and 3 individuals had a $B_{3}$ phenotype. We determined the nucleotide sequences based on PCR followed by subcloning and DNA sequencing of the amplified fragments. Two cases of the $A^{3}$ allele and 1 case of the $B^{3}$ allele were found to contain a single-base substitution which resulted in an amino acid substitution. However, no other cases of $\mathrm{A}^{3}$ and $\mathrm{B}^{3}$ alleles were found to contain differences in this region. This finding demonstrates for the first time heterogeneity among these weak subgroups at the nucleotide level.
\end{abstract}

\section{Introduction}

Since the discovery of the histo-blood group ABO system by Landsteiner in 1900 [1], almost a century passed until we were successfully able to elucidate the molecular genetic basis of the three major alleles $\left(\mathrm{A}^{1}, \mathrm{~B}\right.$, and $\left.\mathrm{O}\right)$ at the blood group $\mathrm{ABO}$ gene locus by cloning $\mathrm{A}^{\mathrm{l}}$ transferase cDNA [2], and by cloning $B$ and $O$ alleles [3] followed by nuclcotide sequencing. $\mathrm{O}$ alleles are characterized by the presence of a single-base delction close to the $\mathrm{N}$-terminal of the coding sequence, which shifts the reading frame of codons and results in the translation of an entirely different protein thereafter without any activity of A or B transferases. $\mathrm{A}^{1}$ - and $\mathrm{B}$-allelic cDNAs are identical except for seven nucleotide substitutions resulting in four amino acid substitutions between $\mathrm{A}^{\mathrm{A}}$ and $\mathrm{B}$ transferases.
We have subsequently extended our study to subgroups. Subgroups of $A, A_{1}$ and $A_{2}$ were first identified by von Dungern in 1911 when he found two kinds of group $A$ erythrocytes. Sera from blood group B individuals contain two types of antibodies against $A$ antigens: anti-A and anti- $A_{1}$. Group A erythrocytes, which react with both anti-A and anti- $A_{1}$ were named $A_{1}$, and those which react only with anti-A, were named $\mathrm{A}_{2}$. Dolichos biflorus lectin has been used as an anti- $A_{1}$ reagent. We analyzed $A^{1} / A^{2}$ differences at the nucleotide level and found a single-base substitution and a single-base deletion in the coding sequence of the last coding exon of $\mathrm{A}^{2}$ alleles [4]. These two differences were found present in all the $\mathrm{A}^{2}$ alleles tested ( 8 specimens). The single-base delction is located close to the carboxyl terminal of the transferase in the coding sequence. As a result of frame shifting, $A^{2}$ transferase has an
Fumi-ichiro Yamamoto, $\mathrm{PhD}$

Department of Molecular Biology

The Biomembrane Institute and

Department of Patholiology. University of Washington

201 Elliot Avenue West. Seattle, WA 98119 (USA)
(9) $1993 \mathrm{~S}$. Karger AG, Basel $0042-9007 / 93 / 0642-01116$ $\$ 2.75 / 0$ 
additional domain composed of 21 amino acids. We constructed an $\mathrm{A}^{2}$ transferase expression construct and performed DNA transfection experiments. We found this single-nucleotide deletion to be responsible for the weak A transferase activity in $\mathrm{A}^{2}$ transferase.

In addition to $A_{2}$, there are other rare subgroups with weaker serological reactivity compared to $A_{1}$. These can be serologically differentiated and include $A_{3}, A_{x}, A_{\text {end }}$, and $A_{\mathrm{c}}$. Similarly, $B$ phenotypes with weaker than normal $B$ activity are designated as $B_{3}, B_{x}, B_{m}, B_{e l}$. These weak subgroups are of great importance because they allow us to characterize their specific glycosyltransferase by studying the mutations in these alleles. $A_{3}$ erythrocytes are characterized by a mixed-field pattern of agglutination with anti-A or anti-A,B reagents. $B_{3}$, the counterpart of $A_{3}$ in $B$, is similarly characterized by a mixed-field agglutination of erythrocytes with anti-B or anti-A,B reagents. Because roughly two thirds of the red cells are agglutinated, $A_{3}$ and $B_{3}$ are also called $A_{60}$ and $B_{60}$, respectively [5].

Here we report the identification of mutations in the nucleotide sequences of the coding region in some cases of $\mathrm{A}^{3}$ and $\mathrm{B}^{3}$ alleles.

\section{Materials and Methods}

\section{Blood Cirouping}

We have analyzed a total of 7 cases of weak subgroups; one $A_{1} B_{3}$, two $A_{3}$, two $A_{3} B$, two $B_{3}$. Blood groups were determined using immunological methods. One case $\left(A_{1} B_{3}\right)$ was confirmed to posses a $B^{3}$ allele through the grouping of its family members.

\section{PCR Amplification}

Genomic DNA was prepared from blood specimens by Proteinase K-SDS method [6]. Reagents for PCR [7] reaction were purchased from Perkin-Elmer Cetus. The pairs of synthetic oligodeoxynucleotides used for PCR are as follows:

fy-121, CGGAATTCCATGTGACCGCACGCCT and fy-122, CGGAATTCTCTACCCTCGGCCACC:

fy-101, CGCAATTCCCGTCCGCCTGCCTTGCAG and

fy-113, CGCiAATTCACCTCTTGCACCGACCC; and fy-112. CGGAATTCCGTTCTGCTAAAACCAAG and fy-114. CCTGGTGTGCGTGGAC, or

fy-127, CGGAATTCCTGGTGTGCGTGGAC.

The oligodeoxynucleotide fy-127 is essentially identical to fy-114 except for the additional $E c o$ RI site at the $5^{\prime}$ end. All the Eco RI sites are artifical in order to facilitate the subsequent subcloning. The reaction mixture was denatured for $5 \mathrm{~min}$ at $95^{\circ} \mathrm{C}$ followed by 45 cycles of $30 \mathrm{~s}$ at $94^{\circ} \mathrm{C}, 30 \mathrm{~s}$ at $55^{\circ} \mathrm{C}$, and $30 \mathrm{~s}$ at $72^{\circ} \mathrm{C}$ in a DNA thermal cycler. DNA was extracted with phenol: chloroform: isoamylalcohol $=25: 24: 1$ and precipitated with ethanol.

Subchoning and DNA Sequencing

After resuspension, DNA was digested with EcoRI, electrophoresed on in agarose gel, and the major fragments with expected size were excised and DNA was extracted with GeneClean kit (Bio 101). Gel-purified DNA was then ligated with either EcoRI-digested, dephosphorylated sequencing vector pT7T3U18 (Pharmacia-LKB) (fy-121-fy-122, fy-101-fy-113, and fy-112-fy-127), or the same vector double digested with EcoRI and SmaI (fy-112-fy-114). Ligated DNA was used to transform XL1-blue strain of Escherichia coli. Plasmid DNA was prepared from a multiple number of transformants, analyzed for the insert, denatured, and used for nucleotide sequencing by the Sanger method [8] with Sequenase (United States Biochemical).

\section{Results}

Figure 1 shows the schematic comparison of the nucleotide and the deduced amino acid sequences of each allele from 7 individuals showing phenotypes of weak subgroups, $A_{3}$ or $B_{3}$, with those of $A^{\prime}, O$, and $B$ alleles.

Two pairs of oligo primers, fy-101 \& fy-113 and fy-112 \& fy-114/fy-127, were used to amplify the coding sequence in the last coding exon. The corresponding sequence for primer fy-101 resides in the intron preceding this last coding exon (the last base of fy-101 is located just adjacent to the splicing acceptor site), and that of fy-112 resides in the $3^{\prime}$ untranslated region (the last base of fy-112 is located 53 bases away from the termination codon on the $3^{\prime}$ side). Primers $f y-113$ and $f y-114 / f y-127$ are homologous to the sequences in the last exon except for the artifical EcoRI sequences of fy-113 and fy-127, and the amplified fragments from these pairs of oligos overlap one another. The third pair of oligos was used to amplify the preceding exon. The corresponding sequences for fy-121 and fy-122 reside in the introns preceding and following this exon, respectively. The last base of fy-121 is located 16 bases away from the $5^{\prime}$ side of the splicing acceptor site and that of fy- 122 is located 11 bases away from the $3^{\prime}$ side of the splicing donor site. Nucleotide sequencing of multiple (more than 10) clones of transformants, transformed with each PCR-amplified fragment, was performed to discriminate between the two alleles on the two homologous chromosomes as well as the occasional errors introduced by Taq DNA polymerase.

We were able to discriminate the two alleles on homologous chromosome pairs in 5 out of the 7 samples tested, based on differences in the nucleotide sequences. However, even with our extensive sequencing we were unable to discriminate the two homologously paired alleles in the remaining 2 cases (both with a phenotype of $A_{3}$ ) in the coding sequence of the last coding exon. More than twenty transformant clones showed an identical sequence suggesting that these $\mathrm{A}^{3}$ alleles are identical to $\mathrm{A}^{1}$ alleles in this region. Figure 1 shows this conclusion. There appears 
Fig. 1. Schematic comparison of the nucleotide and the deduced amino acid sequences of each allele from individuals showing weak subgroup phenotypes, $A_{3}$ or $B_{3}$, with those of $A^{1}, O$, and $B$ alleles. The $A$ residue and the methionine residue of the initiation codon of $\mathrm{A}^{1}$ transferase are numbered 1 (because of the deletion of the three nucleotides in FY-59-5 clone, the numbering is different from that of FY-59-5 presented in reference [2]. Because only the last two coding exons were analyzed for $\mathrm{A}^{3}$ and $\mathrm{B}^{3}$ alleles, only the sequences in this region are compared and the differences are indicated. For each specimen, the two alleles which made up homologous chromosome pairs were discriminated from one another by differences in the nucleotide sequences. For the two $\mathrm{A}^{3} \mathrm{O}$ individuals, we were unable to discriminate between the two homologously parred alleles in the last coding exon. We assume these $A^{7}$ alleles are identical to $\mathrm{A}^{+}$alleles in the last coding exon because no differences were found among more than twenty transformant clones. The figure shows this conclusion. The hatched rectangle in $\mathrm{O}$ alleles indicates the entirely different deduced amino acid sequence due to frame shifting caused by the single-base deletion. The arrow shows the N-terminal of the soluble form of $A^{\prime}$ transferase.

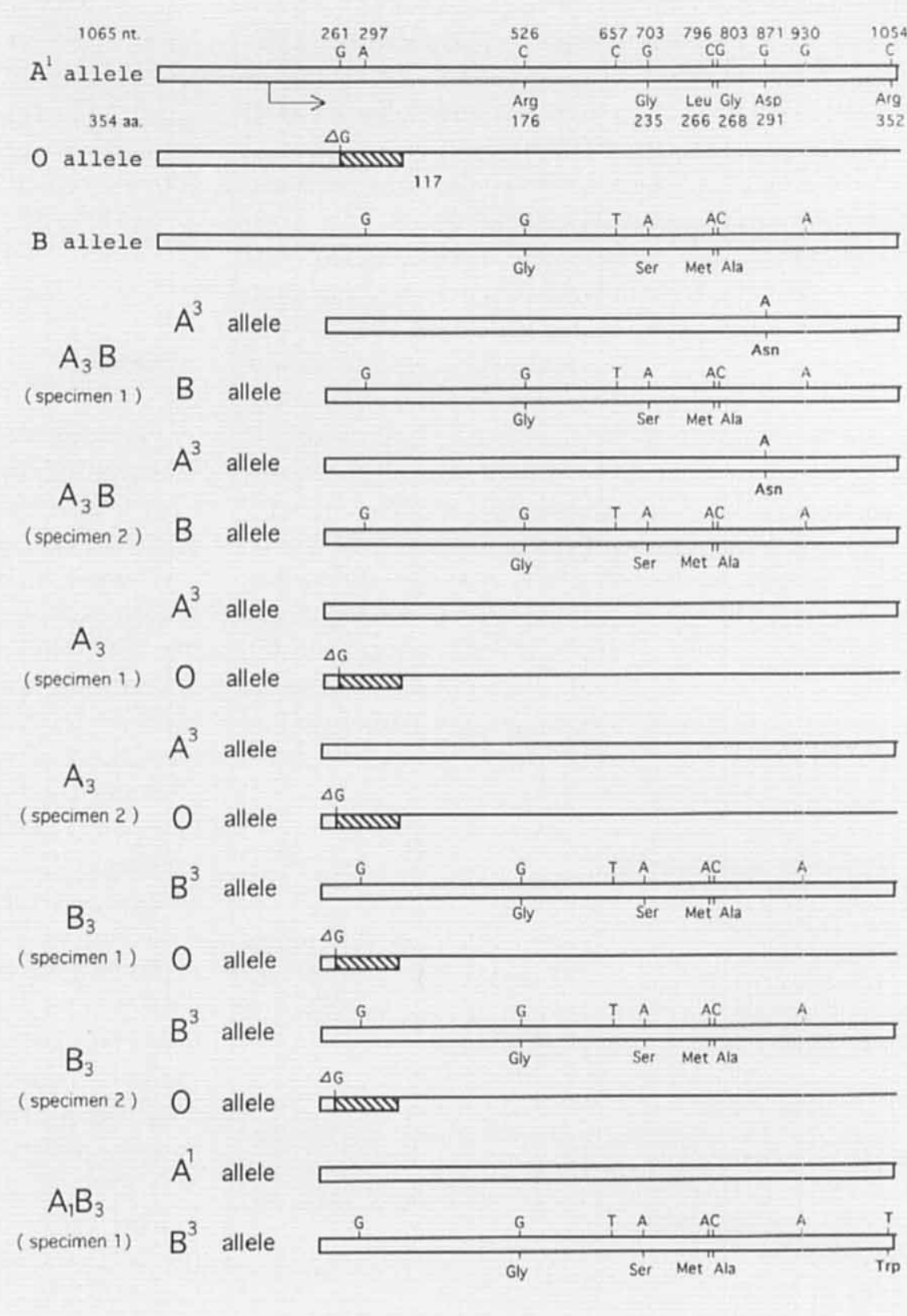

\section{Discussion}

to be at least two kinds of $A^{3}$ and $B^{3}$ alleles. The $A^{3}$ alleles from two $\mathrm{A}_{3} \mathrm{~B}$ individuals have a single-base substitution at nt. $871(\mathrm{G} \rightarrow \mathrm{A})$ resulting in an amino acid substitution at aa. 291 (aspartic acid $\rightarrow$ asparagine) which discriminates these $A^{3}$ alleles from $A^{1}$ alleles. The $B^{3}$ allele from one $A_{1} B_{3}$ individual has a single-base substitution at nt. $1054(\mathrm{C} \rightarrow \mathrm{T})$ resulting in an amino acid substitution at aa. 352 (arginine $\rightarrow$ tryptophan), which discriminates this $B^{3}$ allele from $B$ alleles.
Heterogeneity among the single subgroups has been reported for $A$ and $B$ variants based on the analysis of transferase activity in the serum of these individuals. Cartron et al. [9] reported three types of $A_{3}$ phenotypes. One group consisted of $\mathrm{A}_{3}$ phenotypes with a low level of $\mathrm{A}$ transferase activity in the serum, and the optimal $\mathrm{pH}$ of the transferase was approximately 7 (in 7 out of 11 cases). The second group consisted of $\mathrm{A}_{3}$ phenotypes with no activity of $A$ transferase ( 3 out of the 11). A high A trans- 
ferase activity optimum at $\mathrm{pH} 6$ was detected in the last of eleven $\mathrm{A}$; samples. Nakamura et al. [10] reported an active A transferase which has characteristics (the level of activity, $\mathrm{pH}$ optimum, $\mathrm{K}_{\mathrm{m}}, \mathrm{V}_{\max }$, and heat stability) identical to those of $\mathrm{A}^{\prime}$ transferase in the serum of $\mathrm{A}_{3}$ phenotypes. These authors postulated that the weak A expression is due to the suppression of A transferase production only in hemopoietic tissues. $B$ transferase activity in the serum of individuals with $B_{3}$ phenotype has been reported to vary from 10 to $100 \%$ of control level [11]. Due to the restricted availability of blood specimens, we have not yet performed any enzymological analyses.

Because of our incomplete knowledge of the exon-intron boundaries of $\mathrm{ABO}$ genes and the restricted availability of sample specimens, we focused our efforts on the identification of differences in the coding sequence of the last two coding exons. Although there may be more differences somewhere outside of these exons (including differences in the splicing pattern), $91 \%$ of the soluble form of $\mathrm{A}^{\mathrm{l}}$ transferase is contained in these two exons and extensive nucleotide and deduced amino acid sequence homologies with $\alpha 1 \rightarrow 3$ galactosyltransferase gene are observed only in this region [12] suggesting the importance of the last two coding exons for transferase activity.

We have identified nucleotide substitutions, which result in amino acid substitutions, in the last coding exon of some of $\mathrm{A}^{3}$ and $\mathrm{B}^{3}$ alleles. The fact that these differences were not found common to all the samples with the same weak subgroups indicates heterogeneity in each group.
There is a possibility that only those cases which identify differences in the nucleotide sequence are genuine weak subgroups and the rest are caused by changes in physical conditions due to illness, diet etc. A decrease of A and B antigens in patients with leukemia has been well documented [13].

The computational calculation using the IBI Pustell Sequence Analysis Program indicates the decrease of flexibility around these amino acid substitutions (aspartic acid $\rightarrow$ asparagine at aa. 291 in two $\mathrm{A}^{3}$ alleles and arginine $\rightarrow$ tryptophan at aa. 352 in a $\mathrm{B}^{3}$ allele) as well as the changes in the isoelectric point. The amino acid substitution at aa. 291 has also been calculated to disrupt the $\alpha$-helix structure around this amino acid. These changes may be responsible for the weak activities of these alleles. Future studies by molecular biological approaches (construction of expression constructs with these nucleotide and resulting amino acid substitutions and expression study by DNA transfection) may clarify whether these changes are sufficient for explaining these weak phenotypes. When the complete exon-intron boundaries and promoter sequences are defined for $\mathrm{ABO}$ genes, mutations in other $\mathrm{A}^{3}$ and $\mathrm{B}^{3}$ alleles will be clearly identified.

\section{Acknowledgement}

We thank Ms. Jennifer Stoeck for editorial assistance. This work was supported by the funds from The Biomembrane Institute.

\section{References}

I Landsteiner K: Zur Kenntnis der antifermentativen, lytischen und agglutinierenden Wirkungen des Blutserums und der Lymphe. Zentralbl Bakteriol 1900;27:357-362.

2 Yamamoto F. Marken J, Tsuji T, White T, Claust n H, Hakomori S: Cloning and characterization of DNA complementary to human UDP-(jalNAc: Fuc $\alpha \rightarrow 2$ Gal $\alpha 1 \rightarrow 3$ GalNAc transfiratse (histo-blood group A transferase) mRNA. J Biol Chem 1990;265:1146-1 is1.

3 Yamamoto F, Clausen H, White T, Marken J, Hakomori S: Molecular genetic basis of the histo-blood group ABO system. Nature 1990; 345:220-233.

4 Yamanolo F, McNeill PD, Hakomori S: Human histo-blood group $\mathrm{A}^{2}$ transferase coded by $A^{2}$ allele, one of the $A$ subtypes, is characterized by a single base deletion in the coding sequence, which results in an additional domain at the carboxyl terminal. Biochem Biophys Res Commun 1992;187:366-374.
5 Issit PD: Applied Blood Group Serology, ed 3. Montgomery Scientific Publications, 1985.

6 Maniatis T, Fritsch EF, Sambrook J: Molecular Cloning: A Laboratory Manual. Cold Spring Harbor, Cold Spring Harbor Laboratory, 1982.

7 Saiki RK, Scharf SJ, Faloona F, Mullis KB, Horn GT, Erlich HA, Arnheim N: Enzymatic amplification of $\beta$-globin genomic sequences and restriction site analysis for diagnosis of sickle cell anemia. Science 1985;230:13501354.

8 Sanger F, Miklen S, Coulson AR: DNA sequencing with chain-terminating inhibitors. Proc Natl Acad Sci USA 1977;74:5463-5467.

9 Cartron JP, Badet J, Mulet C, Salmon CH: Study of the $\alpha-\mathrm{N}$-acetylgalactosaminyltransferase in sera and red cell membranes of human A subgroups. J Immunogenet 1978:5:107-116.
10 Nakamura I, Takizawa $H$, Nishino $K$ : $A_{3}$ phenotype with $\mathrm{A}^{1}$ gene-specified enzyme character in serum. Exp Clin Immunogenet 1989:6: 143-149.

11 Salmon C, Cartron JP, Ronger P: The Human Blood Groups. New York, Masson Publishing USA Inc, 1984.

12 Yamamoto F, Hakomori S: Sugar-nucleotide donor specificity of histo-blood group $A$ and $B$ transferases is based on amino acid substitutions. J Biol Chem 1990;265:19257-19262.

13 Race RR, Sanger R: Blood Groups in Man, ed 6. Oxford, Blackwell Scientific Publications, 1975. 